Review

\title{
Wireless Sensor Networks for monitoring underwater sediment transport
}

\author{
A.J. Watt* ${ }^{*}$ M.R. Phillips, C.E-A. Campbell, I. Wells, S. Hole \\ Faculty of Architecture, Computing and Engineering, University of Wales Trinity Saint David, Heol Ynys, Kings Road, Swansea SA1 8EW, UK
}

\section{H I G H L I G H T S}

- Monitoring sediment transport processes is important for understanding coastal erosion.

- Existing coastal monitoring methods are limited to monitoring the visible beach.

- Wireless Sensor Networks are a promising solution in overcoming this limitation.

- This will enable greater understanding of erosion and the coastal environment.

\section{A R T I C L E I N F O}

\section{Article history:}

Received 7 November 2018

Received in revised form 23 February 2019

Accepted 24 February 2019

Available online 27 February 2019

Editor: Damia Barcelo

\section{Keywords:}

Coastal erosion

Coastal monitoring

Remote sensing

Wireless sensor networks

\section{G R A P H I C A L A B S T R A C T}

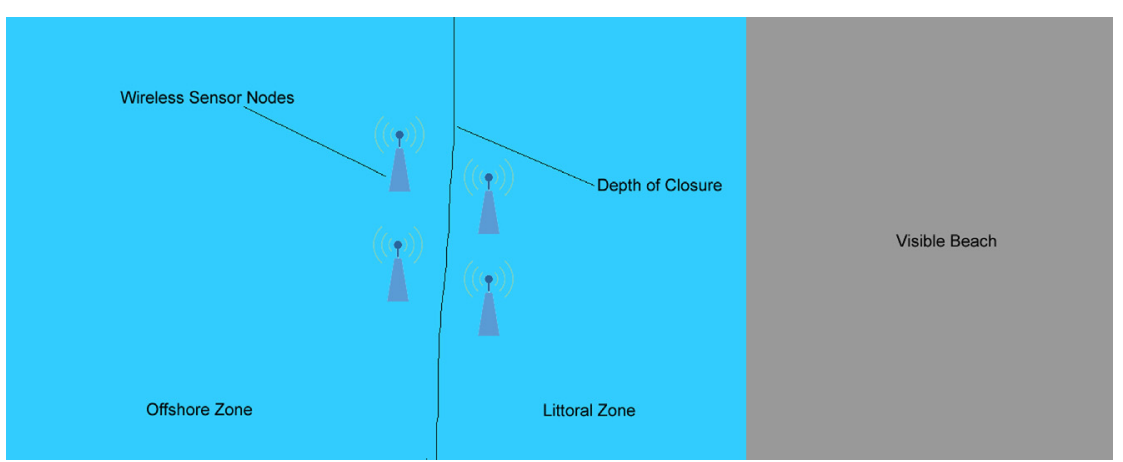

\section{A B S T R A C T}

Monitoring of the coastline and coastal processes, in particular sediment movement, is vital to ensure that erosion response is appropriate given the dynamic nature of coastal systems. This should take place regularly over long periods and it is important that data are collected from submerged portions of the littoral zone, as well as the visible beach. This highlights two limitations in existing coastal monitoring techniques: 1 . they require largely manual operation and 2. are limited to the visible beach, which results in an incomplete picture of what is happening in the coastal zone. Due to the current difficulties in gathering data beneath the sea surface, this paper reviews wireless sensor network (WSN) technology as a means to overcome these limitations. Analysis showed that WSNs are a promising technology for coastal monitoring, not only in terms of overcoming limitations, but also in terms of cost, safety, and the size of areas they are able to monitor. Previous work using WSNs in this environment is somewhat limited, especially as most current methods are largely limited to the visible beach, and do not consider submerged areas of the coastal zone. From consideration of the physical environment, geological and geographical processes, and informed by advances in technology, research gaps are identified, discussed and evaluated to provide strategies for implementation of WSNs to monitor sediment transport.

(c) 2019 Elsevier B.V. All rights reserved.

\section{Contents}

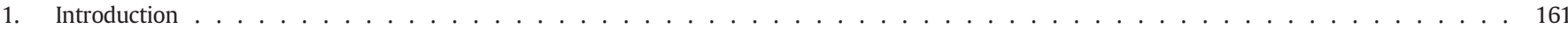

2. Application of Wireless Sensor Networks . . . . . . . . . . . . . . . . . . . . . . . . . . . . . . . 161

\footnotetext{
* Corresponding author.

E-mail address: archie.watt@uwtsd.ac.uk (A.J. Watt).
} 


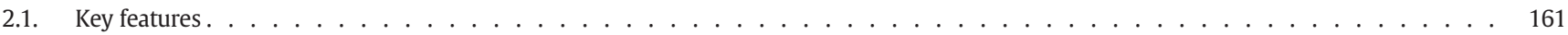

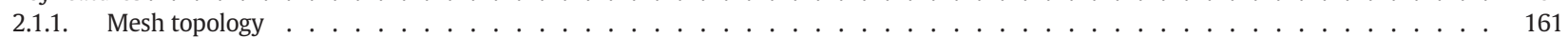

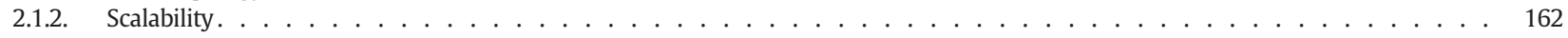

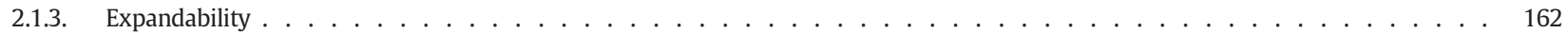

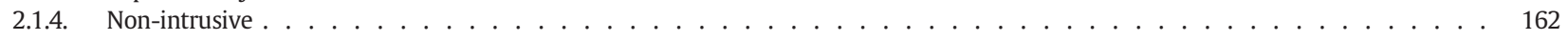

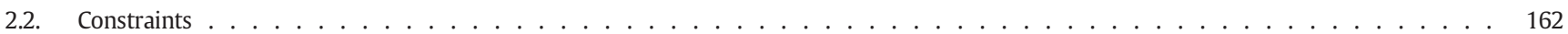

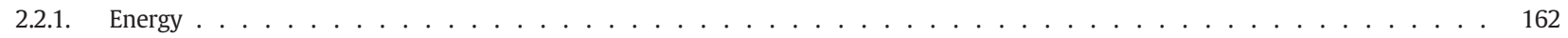

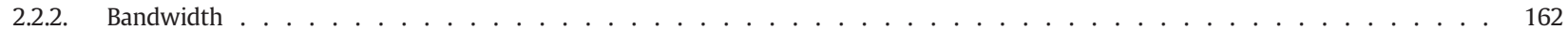

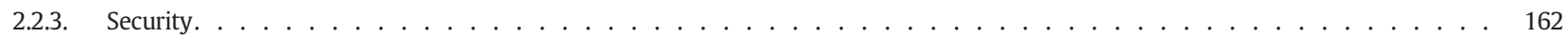

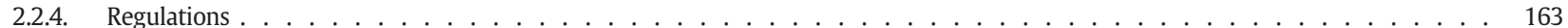

2.2.5. Potential interference . . . . . . . . . . . . . . . . . . . . . . . . . . 163

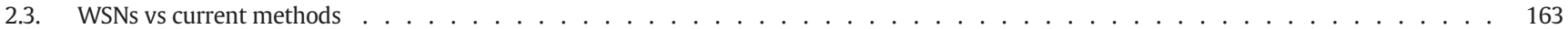

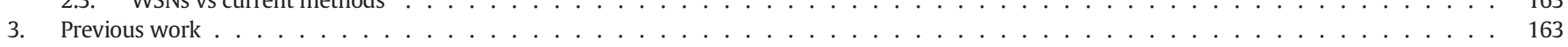

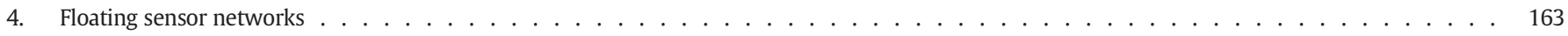

5. Research gaps . . . . . . . . . . . . . . . . . . . . . . . . . . . . . . . . . . . . . . . . . . 164

6. Conclusions and future work. . . . . . . . . . . . . . . . . . . . . . . . . . . . 164

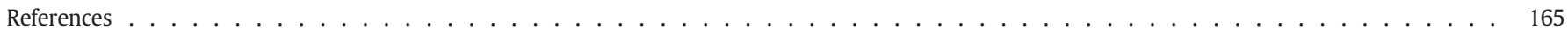

\section{Introduction}

It is widely accepted among climatologists that climate change is an issue that needs to be addressed (Weisse et al., 2014). Although climate change can be both the result of human activity and natural fluctuations, the former, in particular population growth and industrialisation, has been identified as a significant contributor to several climatic events that have occurred in recent years (Pachauri et al., 2014). A recent study by Mann et al. (2016) indicates that 13 out of the 15 warmest years on record would not have occurred if not for the emissions resulting from burning oil and coal.

Climate change has a significant impact on the erosion of sediments on the coastline, primarily through the impact of global warming on sea level rise (SLR). Although coastal erosion, like climate change, is a natural process that has always occurred, human influence, in particular the aforementioned contributions to climate change, as well as urbanisation and economic activities in and around the coast, has resulted in what was once a natural phenomenon becoming an increasingly common and destructive problem of growing intensity, with a 2004 Eurosion report (Salman et al., 2004) highlighting that in Europe, coastal erosion induced by human activity has surpassed that induced by natural factors.

The movement of sediments on coastlines can result in significant erosion or accretion, which can in turn affect important facilities or structures in the area. For example, excessive deposition of sediments may interfere with port and harbour operations, whilst erosion may undermine structures on the coastline. Accordingly, monitoring sediment transport is an essential undertaking in order to collect such data on any coastline where flooding or erosion is occurring or is a potential risk (Phillips, 2005). Given the dynamic nature of coastal systems, this needs to be an ongoing exercise that collects data from both the visible beach and the submerged portions of the littoral zone. Considering the latter requirement, there are significant limitations inherent in current monitoring methods, since they are restricted to monitoring the visible beach. This results in a lack of important data for planning optimal and appropriate responses to coastal erosion. Additionally, operation of current monitoring equipment is largely manual which restricts the frequency with which monitoring activities can be carried out.

The development of an approach to measuring sediment transport beneath the surface of the sea is therefore highly desirable. Considering the limitations identified, the alternative approach would need have two main operational characteristics. Firstly, it must be capable of monitoring the underwater environment. Secondly, it must be able to operate for long periods of time with minimal human intervention.

Wireless Sensor Networks (WSNs) (Khan et al., 2012) are an emerging and fast growing technology, with their growth primarily attributable to the new applications they enable. Specifically, their ability to be deployed in and withstand harsh environments, and to operate with minimal human intervention make them particularly suitable for deployment in areas which are not easily reached. They therefore have significant potential for the application of monitoring sediment transport, and to this end the focus of this paper is to review their suitability for this purpose.

\section{Application of Wireless Sensor Networks}

A Wireless Sensor Network (WSN) (Karl and Willig, 2005; Corke et al., 2010; Oliveira and Rodrigues, 2011; Khan et al., 2012) is a collection of smart devices, otherwise known as sensor "nodes", the numbers of which can range from a few to several hundred or even thousands, depending on the application for which the WSN is deployed. Sensor nodes are typically inexpensive, small, low powered devices, making it easier to deploy them in large quantities.

The sensor nodes in a WSN perform three basic tasks; the collection, processing and transmission of data relating to physical or environmental conditions, for example pressure or temperature. The sensor nodes co-operatively transmit the collected data through the network to its destination, which is normally a "sink" node (also known as a base station), a device capable of performing more complex data processing. Finally, the processed data can be transmitted to a computer where it can then be analysed.

\subsection{Key features}

\subsubsection{Mesh topology}

The nodes in a WSN require the ability to communicate with their neighbours on the network, as well as the sink node which serves as the gateway between the WSN and the final destination. This is especially true when the deployment conditions of the WSN are such that the topology changes regularly, which could occur if additional sensor nodes are regularly added to the network or if sensor nodes are deployed in a location where they are subject to being moved from one location to another (Hossain and Leung, 2007).

If the topology of the network regularly changes, a path through which data is routed on one occasion may not be available on another. As a result, a WSN is typically deployed in the form of a mesh topology (Iyengar and Brooks, 2016), an example of which is depicted in Fig. 1.

This results in the ability of the WSN to route data between sensor nodes by relaying data from node to node until the destination is successfully reached, a technique often referred to as multi-hop communications or multi-hop routing, which allows all nodes in the network to communicate not only with the sink node but also with each other. 


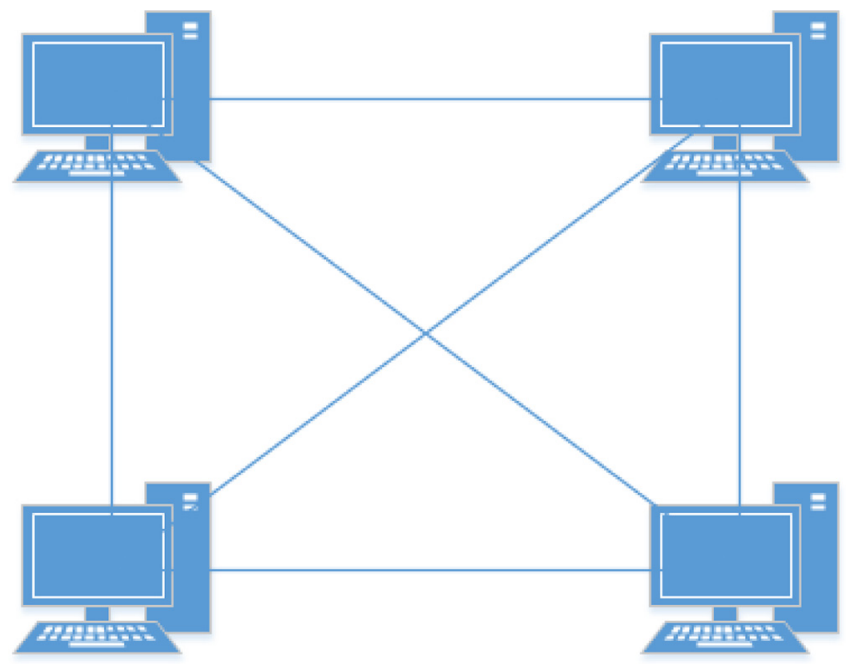

Fig. 1. Mesh topology.

\subsubsection{Scalability}

Scalability refers to the ability of the network to cope with an increasing number of sensor nodes being added to the network over time (Alazzawi et al., 2008). There are two primary features of WSNs that enable them to adapt in response additional nodes being added to the network and the greater volume of traffic that will pass through the network as a result. Firstly, the mesh topology previously discussed in Section 2.1.1 enables new sensor nodes to easily connect to the WSN and configure themselves appropriately. Since all nodes in the network are able to communicate with each other, this allows theoretically unlimited range (Soparia and Bhatt, 2014).

Secondly, sensor networks can be programmed with specialized protocols designed to cope with managing communication between many sensor nodes. The ability of WSNs to scale makes them an advantageous approach when compared to traditional beach monitoring technologies, which are typically only able to monitor one location at once, when in many cases it is highly desirable to be able to monitor multiple simultaneously in order to provide a more complete view of the site and the associated coastal processes.

\subsubsection{Expandability}

Expandability refers to the ability of the nodes in a WSN to have their functionality expanded to include additional features that were not included originally. There are two ways a sensor node can be expanded, which can broadly be defined as software-based expansion and hardware-based expansion. Software based expansion involves programming the node's controller unit to perform additional functions in relation to how the device will respond in specific scenarios to data, whether it be sensor data or network traffic. Hardware-based expansion can be implemented in many ways; for example, expansion to improve power efficiency might involve the addition of solar harvesting capabilities.

In the context of this paper, a WSN may be deployed to determine sediment transport initially, but it may be desirable to later add the ability to monitor other parameters as well, such as temperature or water levels, in order to provide a better range of data on which decisions can be based. As such, a system that takes into account not only the initial requirements, but future requirements as well, is highly advantageous.

\subsubsection{Non-intrusive}

The ability to monitor locations in a non-intrusive manner makes WSNs particularly suitable for monitoring conditions in areas that might be adversely affected by excessive human presence (which could negatively impact the validity of any data obtained). Considering the application of monitoring sediment transport, the non-intrusive aspects of WSNs are particularly beneficial. Most beaches are important recreational resources (Lucrezi et al., 2015), and therefore an intrusive or obstructive method of data collection would likely have adverse effects in terms of less people visiting the beach. Not only could this impact the local economies as a result of less visitors, but it could potentially also impact the validity of the data, in the event that the processes being monitored are influenced by the activities of beach-goers, or by changes made to the beach to accommodate them (e.g. construction) (Burak et al., 2004; Davenport and Davenport, 2006; Phillips and Jones, 2006). As such, it is highly desirable that coastal monitoring equipment be able to operate in a manner that has as little impact as possible on the coastal environment, both in terms of natural processes and in terms of human activity.

\subsection{Constraints}

\subsubsection{Energy}

Energy efficiency is perhaps the most prevalent challenge in WSN operations, due to the typically finite resources of sensor nodes. Although the other constraints discussed in this section can cause significant problems, all are worsened by the broader problem of limited energy resources.

There are three tasks a sensor node carries out that consume energy: sensing, processing, and transmitting. As sensor nodes are usually battery-powered, available node energy is what determines the lifetime and ultimately usefulness of a WSN, and therefore optimizing energy consumption is a major area of WSN-related research (Anastasi et al., 2009; Rault et al., 2014). In many WSN deployments, such as those underwater (Heidemann et al., 2006), replacing batteries is often impractical, in some cases impossible, and the WSN is effectively rendered useless when the batteries are depleted. As such, efficient energy consumption, whilst important in any deployment, must be a crucial consideration of designing any battery-powered WSN that is deployed in a location where energy cannot easily be replenished.

\subsubsection{Bandwidth}

A number of constraints are present in wireless networks as a result of the available bandwidth being shared between all devices that are within radio range of each other, since they all make use of the same transmission medium. Node density, although a benefit in terms of enabling multi-hop routing, may also be a drawback in terms of bandwidth availability, since the greater the node density, the less bandwidth will be available for each individual node. This issue is compounded by the fact that sensor nodes generally only have rather small bandwidth amounts of bandwidth to utilize to begin with, due to the energy constraints that were discussed Section 2.2.1. Therefore, whilst the bandwidth of most typical wireless local area networks (WLANs) can be measured in megabits-per-second ( $\mathrm{mbps}$ ), WSN bandwidth is generally measured in the order of kilobits-per-second (kbps). With this in mind, it is crucial to utilize available bandwidth as effectively as possible.

\subsubsection{Security}

Security is an important concern in any computer network, though generally more in those that use wireless communication, since no physical connection is required for an attacker to gain access. Whilst the attacks that a WSN is vulnerable to are much the same as any other wireless network, the problems are often exacerbated by the broader problem of energy limitations, since the more complex a security protocol the more processing work a sensor node will need to do. As a result, it may be necessary to make a compromise between security and energy efficiency; which takes priority will likely depend on the specific application of the WSN. If sensitive data is being collected (for example in the case of military deployments), it might require 
encryption to prevent it being read in the event it was compromised. A detailed discussion of WSN security is provided in (Chen et al., 2009).

\subsubsection{Regulations}

The regulations that apply to the design, deployment and monitoring of devices in the coastal environment vary by country. In the United Kingdom, many activities in the marine environment require a licence, applications for which are considered according to the UK Marine Policy Statement (UK Government, 2011). The process of obtaining licences can be lengthy depending on the information that is required as part of the application process. For example, data on sediment analysis may be required in support of an application, usually from within the last three years (Marine Management Organisation, 2014). If such data does not exist then the necessary sampling and analysis of sediments must be carried out before an application can be submitted, delaying the process. As such, the Marine Management Organisation (MMO) recommends consulting with them prior to submitting an application, to ensure that requirements are understood in advance.

The Marine and Coastal Access Act (UK Parliament, 2009) specifies several activities that are licensable, one of which is the construction, alteration or improvement of any works either in or over the sea or on or under the sea bed, a category WSN deployments would fall under. The basic regulatory expectations for floating marine devices are outlined in a recent report from the Maritime and Coastguard Agency (2017), in terms of design, hardware, installation, monitoring, and verification.

This demonstrates the importance of considering relevant regulation not just at the deployment stage, but during the initial design and construction stages as well. Therefore, consultation with the relevant regulation and associated organisations must occur from first principles right through to deployment.

\subsubsection{Potential interference}

Given the nature of the coastal environment, there is significant potential for deployed monitoring devices to be interfered with, whether it be by natural forces (e.g. wind/waves), wildlife, or human activities (e.g. boats/diving). The regulations that were previously discussed in Section 2.2.4 are largely informed by the potential interference that could occur; for example, stipulating that the hardware utilised must be certified to be of an appropriate standard and quality, and the requirements that the device have the ability to be continuously monitored so that an alert could be sent in the event of interference or failure (Maritime and Coastguard Agency, 2017). Consequently, ensuring compliance with the relevant regulations can contribute significantly to alleviating the risks of interference.

Certain characteristics of WSNs also contribute to alleviating this issue. In particular, their ability to monitor locations in a non-intrusive way (as discussed in Section 2.1.4), make them less susceptible to interference because they are less likely to, for example, attract wildlife. Additionally, sensor nodes are typically low-cost devices, so in the event of a device failure due to interference, the cost of replacing the device would be minimised (Oliveira and Rodrigues, 2011).

\subsection{WSNs vs current methods}

The monitoring of the coastal zone is generally achieved by means of a small number of expensive and high precision sensing devices, which collect the relevant data which must then be manually downloaded and analysed, although some such devices can be combined with long-range communication networks, which has the benefit of allowing locations to be monitored remotely (Oliveira and Rodrigues, 2011). This paper has discussed in detail WSNs as a means to improve monitoring technologies, giving particular consideration to the limitations identified with current methods that were stated in Section 1. Compared to traditional monitoring methods, WSN technology has several advantages, which are discussed in the remainder of this section.
Firstly, WSNs can be deployed in locations that may not be reachable by researchers, for example underwater, thus enabling a better range of data to be collected and a more complete understanding of a process, such as erosion, to be established. Furthermore, since they can be deployed permanently, they are particularly advantageous for the monitoring of areas where repeated visits would be potentially unsafe for researchers.

In terms of reliability, WSNs are typically deployed in the form of a mesh topology (Groth et al., 2005), in which all nodes are able to communicate directly with each other, eliminating the single point of failure that exists with traditional technologies. Additionally, the multi-hop nature of this topology enables potentially unlimited communication range, as more sensor nodes can be added to the network as required.

Finally, current coastal monitoring technologies, whilst providing real-time data, typically require the researcher to be present to operate the device and take measurements, with future measurements requiring future visits. A WSN provides substantial economic benefits by comparison, since they enable data to be accessed in real-time without repeated visits to the monitored site being necessary. Furthermore, logistics are greatly reduced to deployment of the network and occasional maintenance (Polastre et al., 2004).

\section{Previous work}

The application of WSNs to monitoring sediment transport has been investigated in a number of previous studies, a recent example being the work of Pozzebon et al. (2018). However, there is limited literature available in this area, and much like traditional techniques, these studies have been largely limited to monitoring the visible beach, without consideration for the submerged region of the coastal zone.

Examples of WSN technology being applied to underwater sediment transport are even more scarce. The primary example is previous work also carried out by the University of Wales Trinity Saint David (UWTSD), which developed an underwater sensor network that deployed differential pressure sensors on the seabed (Che et al., 2009), known commercially as Automated Sensing Technologies for Coastal Monitoring (ASTEC). The sensor nodes were fixed in position and measured the movement of sediment by determining the amount of sediment that was settling on top of them. Data was gathered at two-hour intervals and transferred to the sink node on the surface once a day. The sink node would then transmit the collected data via the mobile phone network to a computer for analysis (Wells et al., 2009).

The ASTEC project was successfully deployed and demonstrated proof-of-concept, however, little useful data could be gathered due to problems relating to energy consumption and associated issues with deployment and maintenance. Our previous work (Watt et al., 2016) therefore carried out a comparison between submerged and floating WSN deployments for the purpose of monitoring underwater sediment transport, which highlighted a number of advantages to using a floating deployment. The findings of this are summarised in Table 1.

\section{Floating sensor networks}

Floating sensor networks combine echo sounding, the most common means of measuring ocean depth, with WSN technology, which in combination with wave statistics and bathymetric data enable the movement of sediments to be determined. In common with most coastal monitoring programmes, the initial stage of applying this method involves the establishment of a datum, a baseline measurement against which future change can be measured. This should include correlation with wave statistics and bathymetric data in order to take wave motion and the seabed's topographical features into account.

Depth of closure (DoC) is the depth at which there is little or no motion of sediments and accordingly, it is a point at which there would be an accretion of sediments due to their lack of motion (Kraus et al., 1998). Therefore, a depth measurement taken at this point would be smaller 
Table 1

Floating vs submerged deployments.

\begin{tabular}{|c|c|c|}
\hline Criteria & $\begin{array}{l}\text { Underwater sensor } \\
\text { networks }\end{array}$ & $\begin{array}{l}\text { Floating sensor } \\
\text { networks }\end{array}$ \\
\hline Cost & $\begin{array}{l}\text {-Complex node design } \\
\text {-Advanced hardware } \\
\text { protection }\end{array}$ & $\begin{array}{l}\text { Less complexity } \\
\text { required in transceiver } \\
\text { design and node } \\
\text { processing }\end{array}$ \\
\hline Deployment/maintenance & $\begin{array}{l}\text {-Heavy and bulky due to } \\
\text { hardware protection } \\
\text {-Difficult to maintain once } \\
\text { deployed }\end{array}$ & $\begin{array}{l}\text { Relatively easy both to } \\
\text { deploy and retrieve for } \\
\text { maintenance purposes. }\end{array}$ \\
\hline Medium & $\begin{array}{l}\text { No optimal communication } \\
\text { medium }\end{array}$ & $\begin{array}{l}\text {-No underwater } \\
\text { communication } \\
\text { necessary } \\
\text {-ZigBee, 6LoWPAN can } \\
\text { be used }\end{array}$ \\
\hline Power supply & $\begin{array}{l}\text {-Complex DSP resulting in } \\
\text { higher power requirements } \\
\text {-Difficult to replenish } \\
\text { power }\end{array}$ & $\begin{array}{l}\text {-Reduced } \\
\text { communication power } \\
\text { requirements } \\
\text {-Easier to } \\
\text { replace/recharge power } \\
\text { supply }\end{array}$ \\
\hline Localization & $\begin{array}{l}\text { No firmly established } \\
\text { method. }\end{array}$ & $\begin{array}{l}\text { Can be used with GPS to } \\
\text { accurately establish the } \\
\text { node's position. }\end{array}$ \\
\hline Security & $\begin{array}{l}\text { Increased power } \\
\text { requirements, less power } \\
\text { available for security } \\
\text { protocols. }\end{array}$ & $\begin{array}{l}\text { Can take advantage of } \\
\text { establish standards that } \\
\text { consider security, such } \\
\text { as ZigBee/6LoWPAN }\end{array}$ \\
\hline
\end{tabular}

than one taken where sediments are still moving. This principle is illustrated in Fig. 2. By comparing this reading with the earlier established baseline, it can be determined whether accretion or erosion is occurring.

\section{Research gaps}

The current state of knowledge surrounding each of the following identified research gaps leaves the application of WSNs for monitoring underwater sediment transport in need of more extensive research efforts. This section presents a summary of research gaps that need to be addressed in future research.

Firstly, there is currently no method of monitoring the movement of sediments beneath the surface of the sea. This results in a lack of important data for coastal engineers to utilize in the development of coastal management strategies. The characteristics of WSN technology make them a very promising solution to the limitations identified with current monitoring methods. However, examples of deploying this technology for this purpose are limited. Whilst there have been recent studies that investigate the use of WSNs for monitoring sediment transport these, much like current methods, have been largely limited to monitoring the visible beach and do not consider underwater activity.

Previous work undertaken by UWTSD (ASTEC) developed an underwater wireless sensor network (UWSN) to monitor sediment transport on the seabed (Che et al., 2009). However, it was not able to collect significant amounts of useful data, due to problems relating to energy consumption as well as more general implications associated with underwater deployments.

Floating WSN deployments have the potential to overcome most of the problems that hindered the usefulness of the ASTEC deployment (Watt et al., 2016). A floating deployment would measure the water depth, and by correlating this with data on tide levels and the topography of the seabed the change in sediment levels could be measured. More research needs to be carried out to determine the feasibility of this approach.

\section{Conclusions and future work}

This paper has discussed the application of WSN technology to monitoring underwater sediment transport, something that is not possible using current monitoring methods. Although WSNs show considerable promise in overcoming the limitations of current methods, the literature showing examples of them being deployed for this application is sparse. This review suggests that there is a need for multi-disciplinary study involving collaboration between the coastal engineering and computer networking disciplines. Based on the research gaps identified in Section 5, our future work will focus upon the development of a novel floating wireless sensor network capable of measuring the movement of sediment. Initial testing will be undertaken in a laboratory environment, following which more extensive field trials can be undertaken in order to test this approach in a real-world environment. Establishing a method of monitoring the movement of sediments underwater, rather than just on the visible beach, will enable coastal monitoring programmes to better understand the coastal environment and the

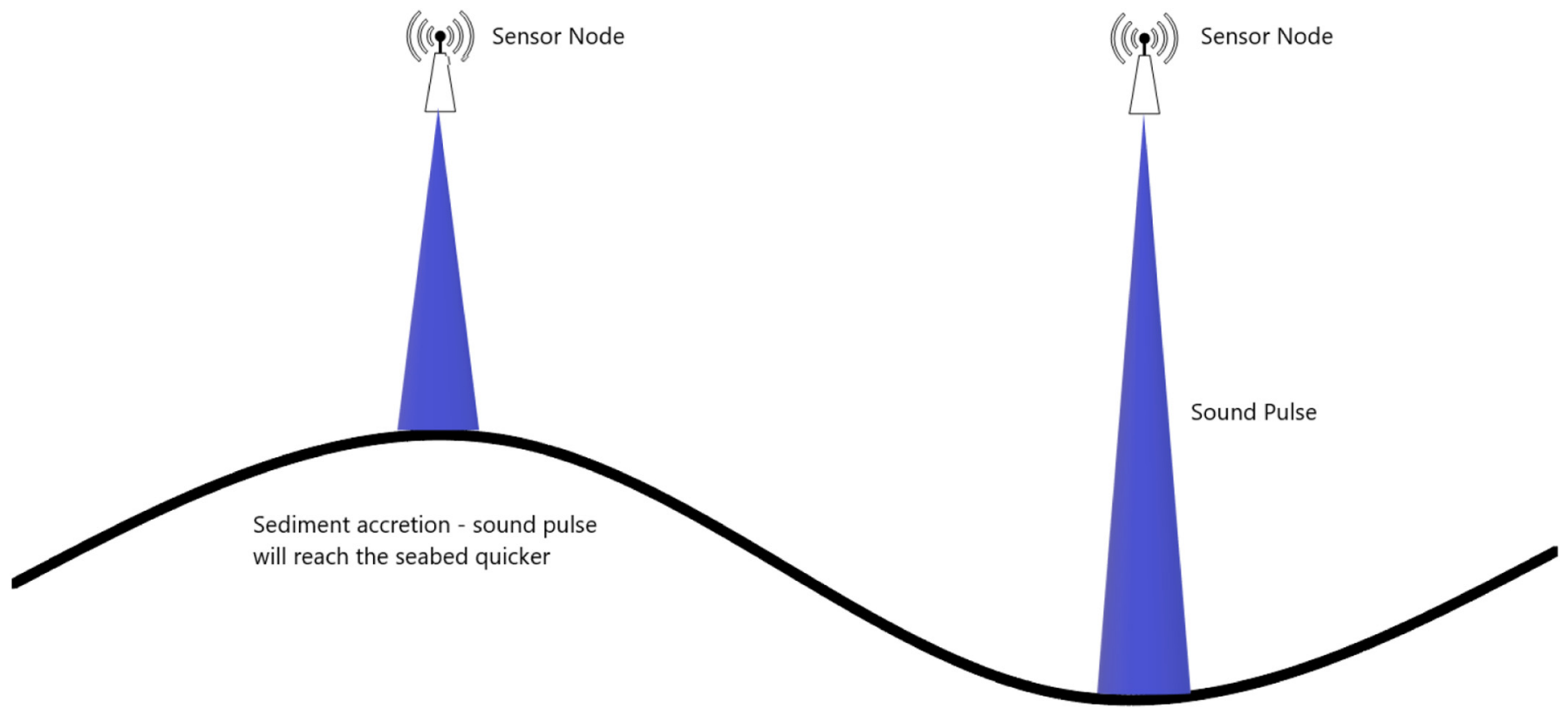

Fig. 2. Floating sensor network. 
associated processes, and in turn contribute to the development of more effective coastal management strategies.

\section{References}

Alazzawi, L.K., et al., 2008. Scalability analysis for Wireless Sensor Networks routing protocols. 22nd International Conference on Advanced Information Networking and Applications - Workshops (AINA workshops 2008). IEEE, pp. 139-144 https://doi.org/ 10.1109/WAINA.2008.178.

Anastasi, G., et al., 2009. Energy Conservation in Wireless Sensor Networks: A Survey. 7 (3). Ad Hoc Networks. Elsevier B.V, pp. 537-568. https://doi.org/10.1016/j. adhoc.2008.06.003.

Burak, S., Dogan, E., Gazioglu, C., 2004. Impact of urbanization and tourism on coastal environment. Ocean \& Coastal Management 47 (9-10), 515-527. https://doi.org/ 10.1016/j.ocecoaman.2004.07.007.

Che, X., et al., 2009. A static multi-hop underwater wireless sensor network using RF electromagnetic communications. Icdcs: 2009 International Conference on Distributed Computing Systems Workshops, pp. 460-463 https://doi.org/10.1109/ ICDCSW.2009.36.

Chen, X., et al., 2009. Sensor network security: a survey. IEEE Commun. Surv. Tutorials 11 (2), 52-73. https://doi.org/10.1109/SURV.2009.090205.

Corke, P., et al., 2010. Environmental wireless sensor networks. Proc. IEEE 98 (11), 1903-1917. https://doi.org/10.1109/JPROC.2010.2068530.

Davenport, J., Davenport, J.L., 2006. The impact of tourism and personal leisure transport on coastal environments: a review. Estuar. Coast. Shelf Sci. 67 (1-2), 280-292. https://doi.org/10.1016/j.ecss.2005.11.026.

Groth, D., et al., 2005. Network+ Study Guide: Exam N10-003. Wiley Available at: https://books.google.co.uk/books?id=eldC-w9SN-0C.

Heidemann, J., Wills, J., Syed, A., 2006. Research challenges and applications for underwater sensor networking. IEEE Wireless Communications and Networking Conference, 2006. WCNC, pp. 228-235 https://doi.org/10.1109/WCNC.2006.1683469.

Hossain, E., Leung, K.K., 2007. Wireless Mesh Networks: Architectures and Protocols Springer, US Available at:. https://books.google.co.uk/books?id=7oAhcb6Ha94C.

Iyengar, S.S., Brooks, R.R., 2016. Distributed Sensor Networks: Sensor Networking and Applications. Chapman \& Hall/CRC Computer and Information Science Series vol. 2. CRC Press Available at:. https://books.google.co.uk/books?id=ffHRBQAAQBAJ.

Karl, H., Willig, A., 2005. Protocols and Architectures for Wireless Sensor Networks. John Wiley and Sons.

Khan, S., Pathan, A., Alrajeh, N., 2012. Wireless Sensor Networks: Current Status and Future Trends. CRC Press.

Kraus, N., Larson, M., Wise, R., 1998. Depth of closure in beach-fill design. Coastal Engineering Techinical Note, CETN (II-40)

Lucrezi, S., Saayman, M., Van der Merwe, P., 2015. 'Managing Beaches and Beachgoers: Lessons From and for the Blue Flag award', Tourism Management. 48. Elsevier Ltd, pp. 211-230. https://doi.org/10.1016/j.tourman.2014.11.010.
Mann, M.E., et al., 2016. The likelihood of recent record warmth. Sci. Rep. 6 (1), 19831. https://doi.org/10.1038/srep19831.

Marine Management Organisation, 2014. Marine licensing: sediment analysis and sample plans. Available at:. https://www.gov.uk/guidance/marine-licensing-sediment-analysis-and-sample-plans, Accessed date: 2 January 2019.

Maritime \& Coastguard Agency, 2017. Regulatory expectations on moorings for floating wind and marine devices. Available at. https://assets.publishing.service.gov.uk/government/uploads/system/uploads/attachment_data/file/640962/Regulatory_expectations_on_mooring_devices_from_HSE_and_MCA.PDF.

Oliveira, L.M., Rodrigues, J.J.P., 2011. Wireless sensor networks: a survey on environment monitoring. J. Commun. 6 (2), 143-151. https://doi.org/10.4304/jcm.6.2.143-151.

Pachauri, R.K., et al., 2014. In: Pachauri, R.K., Meyer, L. (Eds.), Climate Change 2014: Synthesis Report. Contribution of Working Groups I, II and III to the Fifth Assessment Report of the Intergovernmental Panel on Climate Change. IPCC, Geneva, Switzerland.

Phillips, M.R., 2005. An Assessment of Processes and Strategies for Management of the Penarth Coast. PhD Thesis. Bath Spa University.

Phillips, M.R., Jones, A.L., 2006. Erosion and tourism infrastructure in the coastal zone: problems, consequences and management. Tour. Manag. 27 (3), 517-524. https:// doi.org/10.1016/j.tourman.2005.10.019.

Polastre, J., et al., 2004. Analysis of Wireless Sensor Networks for habitat monitoring. Wireless Sensor Networks. Springer US, Boston, MA, pp. 399-423 https://doi.org/ 10.1007/978-1-4020-7884-2_18.

Pozzebon, A., et al., 2018. A wireless sensor network for the real-time remote measurement of aeolian sand transport on sandy beaches and dunes. Sensors 18 (3), 820. https://doi.org/10.3390/s18030820.

Rault, T., Bouabdallah, A., Challal, Y., 2014. Energy efficiency in wireless sensor networks: a top-down survey. Computer Networks. 67. Elsevier B.V., pp. 104-122. https://doi. org/10.1016/j.comnet.2014.03.027.

Salman, A., Lombardo, S., Doody, P., 2004. Living With Coastal Erosion in Europe: Sediment and Space for Sustainability.

Soparia, J., Bhatt, N., 2014. A survey on comparative study of wireless sensor network topologies. Int. J. Comput. Appl. 87 (1), 40-43. https://doi.org/10.5120/15175-3255.

UK Government, 2011. UK marine policy statement. Available at:. https://assets.publishing.service.gov.uk/government/uploads/system/uploads/attachment_data/file/ 69322/pb3654-marine-policy-statement-110316.pdf.

UK Parliament, 2009. Marine and Coastal Access Act. UK Parliament Available at:. https:// www.legislation.gov.uk/ukpga/2009/23/contents.

Watt, A.J., et al., 2016. A comparative assessment of floating and submerged sensor network deployments for monitoring underwater sediment transport processes. J. Comput. Commun. 04 (05), 41-45. https://doi.org/10.4236/jcc.2016.45006.

Weisse, R., et al., 2014. Changing extreme sea levels along European coasts. Coast. Eng. 87, 4-14. https://doi.org/10.1016/j.coastaleng.2013.10.017.

Wells, I., et al., 2009. Node pattern simulation of an undersea sensor network using RF electromagnetic communications. 2009 International Conference on Ultra Modern Telecommunications \& Workshops. IEEE, pp. 1-4 https://doi.org/10.1109/ ICUMT.2009.5345555 